\title{
Synthesis and Characterization of Resorcinarene-Encapsulated Nanoparticles
}

Alexander Wei, ${ }^{*}$ Kevin B. Stavens, ${ }^{\S}$ Stephen V. Pusztay, ${ }^{*}$ Ronald P. Andres ${ }^{\S}$

"Department of Chemistry, Purdue University, West Lafayette, IN 47907 (alexwei@purdue.edu)

${ }^{\S}$ Department of Chemical Engineering, Purdue University, West Lafayette, IN 47907

\begin{abstract}
A new strategy for stabilizing inorganic nanoparticles in nonpolar solutions is described. Resorcinarenes 1-3 were synthesized and evaluated as surfactants because of their large concave headgroups with multiple contact sites. Au nanoparticles ranging from 3-20 nm in diameter were generated in the vapor phase and dispersed into dilute hydrocarbon solutions of 1-3, where they were stabilized for up to several months. Chemisorption is most likely mediated by multiple Au-O interactions, as indicated by several control experiments and by surface-enhanced Raman spectroscopy. The resorcinarenes were readily displaced by dodecanethiol, which resulted in the precipitation of particles $>5 \mathrm{~nm}$ as determined by absorption spectroscopy and transmission electron microscopy. This suggests that the mobility of the resorcinarene tailgroups are important for maintaining the larger nanoparticles in a dispersed state. Resorcinarene surfactants with stronger chemisorptive properties are currently being explored.
\end{abstract}

\section{INTRODUCTION}

Nanoparticle-based devices are being envisioned as the next generation in electronics miniaturization because of their quantized electromagnetic and photonic properties. ${ }^{1}$ However, quantum devices can be expected to be highly sensitive to charge defects, so an important consideration during fabrication is the ability to stabilize and manipulate charge-neutral nanoparticles in a low-dielectric medium. Chemisorptive surfactants such as the alkanethiols have been successful in stabilizing small $(<10 \mathrm{~nm})$ gold nanoparticles against agglomeration in hydrocarbon solutions, but alkanethiol-passivated clusters $>10 \mathrm{~nm}$ do not exhibit long-term stability and precipitate at ambient temperatures. ${ }^{2}$ This behavior is due largely to the rapid increase in the attractive van der Waals force between particles as a function of their size, ${ }^{3}$ but entropic effects can also play an important role. In the case of the alkanethiols the small headgroups encourage a dense packing of the hydrocarbon chains, which promotes flocculation.

Here we address the issue of size-dependent agglomeration by using resorcinol-derived calixarenes (resorcinarenes ${ }^{4}$ ) as nanoparticle surfactants (see Figure 1). These macrocycles are appended by several hydrocarbon tails and are well suited for nanoparticle encapsulation. First, the resorcinarene headgroup is rimmed with multiple oxygen atoms for polydentate binding to the nanoparticle surface. Second, the relatively high surface area of the concave headgroup (ca. $1 \mathrm{~nm}$ diameter) decreases the surfactant/particle ratio and consequently the entropic cost of selfassembly. ${ }^{5}$ Finally, the tailgroups are spaced such that they are highly mobile and do not form tightly packed domains, thereby increasing the solubility of the encapsulated materials. These surfactant properties are demonstrated by stabilizing neutral gold nanoparticles of up to $20 \mathrm{~nm}$ in hydrocarbon solutions.

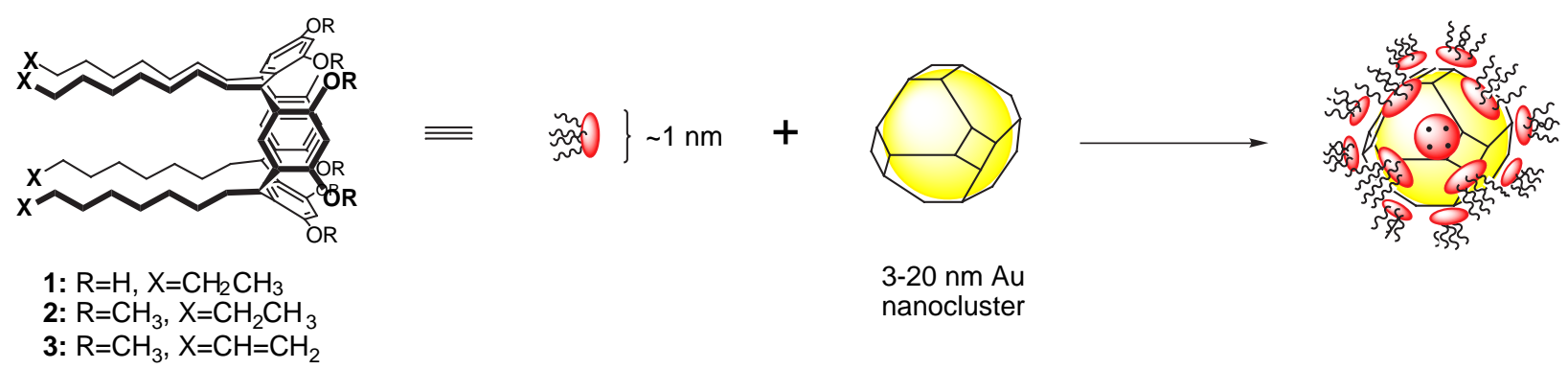

Figure 1. Encapsulation of Au nanoparticles by resorcinarenes 1-3. 


\section{EXPERIMENT AND RESULTS}

Neutral gold nanoparticles were prepared in the gas phase by condensation of pure metal vapor and were captured by passage through surfactant solutions in mesitylene. ${ }^{7}$ Resorcinarenes 1-4 were synthesized according to literature procedures ${ }^{8}$ and were diluted to 0.1-1.2 mM. Aerosols of gold nanoparticles of controlled size ranges were generated using a distributed arc cluster source $(\mathrm{DACS})^{9}$ and bubbled directly into the resorcinarene solutions, which turned deeply purple upon capture. This procedure was noteworthy in at least two respects. First, we noted that the resorcinarenes were several times more efficient at capturing nanoparticles than dodecanethiol $(\mathrm{C} 12 \mathrm{SH})$ at equimolar surfactant concentrations; at $520 \mathrm{~nm}$, the characteristic Mie resonance for isolated gold particles, solutions of $3.7 \pm 1.5 \mathrm{~nm} \mathrm{Au}$ particles captured in $1.2 \mathrm{mM}$ of 1 or 2 absorbed 5-6 times more strongly than solutions of particles captured in $1.2 \mathrm{mM} \mathrm{C12SH}$. Second, both large $(>10 \mathrm{~nm})$ and small $(<10 \mathrm{~nm})$ nanoparticles remained well dispersed in millimolar solutions of $\mathbf{1}$ and $\mathbf{2}$ at ambient temperature over a period of several months, with minimal loss of absorption intensity or precipitation. Transmission electron microscopy (TEM) of nanoparticles captured by 1 revealed a significant proportion of fused particles, suggesting that the kinetics of aggregation were competitive with surfactant passivation (see Figure 2a). It is likely that hydrogenbonded aggregation of $\mathbf{1}$ interferes with adsorption of the headgroups to the nanoparticle surface. In contrast, minimal aggregation was observed among the nanoparticles captured by octamethyl derivative 2 (see Figure $2 \mathrm{~b}$ ). The nanoparticle size distributions did not change over time in either case, indicating that the encapsulated materials were quite stable under these conditions.
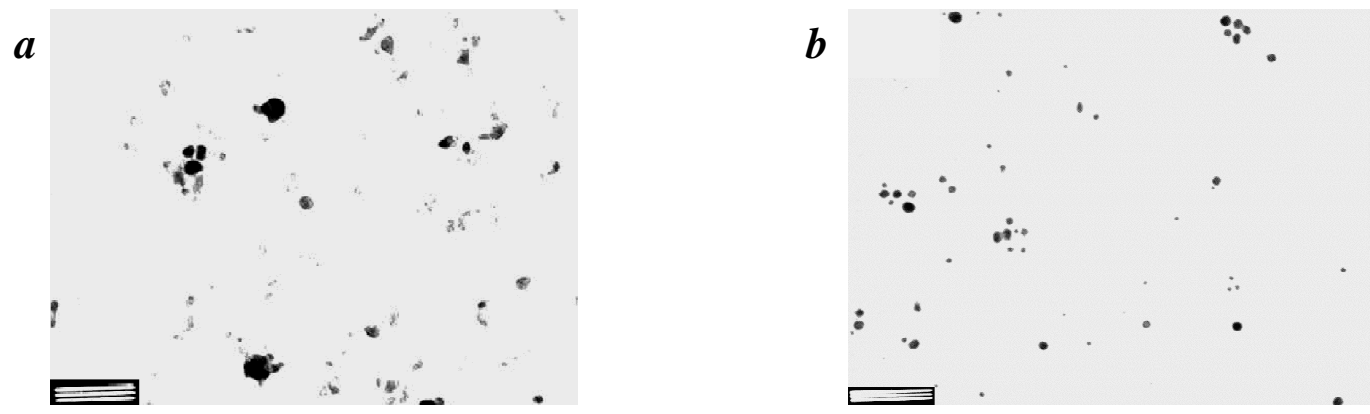

Figure 2. Transmission electron micrographs (Jeol $2000 \mathrm{FX}, 200 \mathrm{KeV}$ ) of Au nanoparticles generated in the gas phase and dispersed into $1.2 \mathrm{mM}$ solutions of resorcinarenes $1(a, l e f t)$ and $2(b, r i g h t)$. Bar $=50 \mathrm{~nm}$.

Stabilization of the Au nanoparticles by the resorcinarene headgroup is most likely mediated by chemisorption via multiple Au-O interactions. The eight oxygen atoms along the rim of the resorcinarene headgroup can adsorb to the Au surface in a cooperative fashion, similar to the ionbinding properties of the macrocyclic polyethers, the prototypical supramolecular receptor. ${ }^{10}$ The enthalpy of Au-O chemisorption is remarkably low, ${ }^{11}$ but its role in nanoparticle stabilization is supported by the observation that tetra- $O, O$-methylene resorcinarene derivative $4,{ }^{8 b}$ whose oxygen lone pairs are prevented from chemisorbing cooperatively due to steric hindrance, completely failed to capture or stabilize Au nanoparticles.

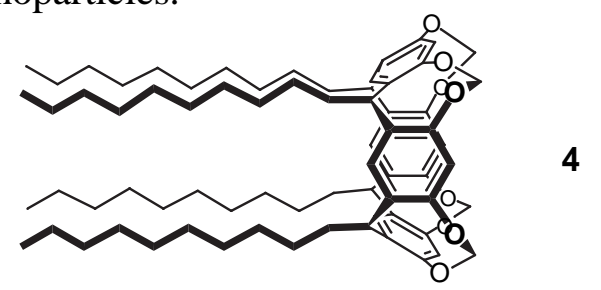

Surface adsorption was characterized by surface-enhanced Raman spectroscopy (SERS), a powerful and highly sensitive method for characterizing monolayer adsorbates on noble metal particles. ${ }^{12}$ Large Au clusters encapsulated by 2 were gently precipitated by addition of one volume of $\mathrm{CH}_{3} \mathrm{CN}$ to remove excess surfactant, then redissolved in mesitylene and drop-coated onto a glass surface. This substrate was observed to produce strong Raman signals (see Figure 3a), which we presume to be generated only by molecules adsorbed onto the nanoparticle surfaces. In 
the absence of nanoparticles, thin films of $\mathbf{2}$ did not generate detectable amounts of Raman backscattering. Although we are presently unable to make an unambiguous assignment of all the group frequencies, there are two pieces of evidence which are strongly suggestive of a specific mode of chemisorption. First, many of the signals in Figure 3a are substantially different in frequency and/or intensity from those produced by polycrystalline samples of pure $\mathbf{2}$ (see Figure $3 \mathrm{~b})$. Second, several of the peaks in Figure $3 \mathrm{a}$ are significantly broadened, most notably those at 720 and $856 \mathrm{~cm}^{-1}\left(\Delta v_{1 / 2} \sim 30 \mathrm{~cm}^{-1}\right)$. This broadening is commonly attributed to inhomogeneities due to chemisorption on different surface lattices or a decrease in vibrational excited-state lifetimes, both of which can strongly affect the bandwidths of the vibrational modes of surfactants which are directly adsorbed onto the metal surface. ${ }^{13}$
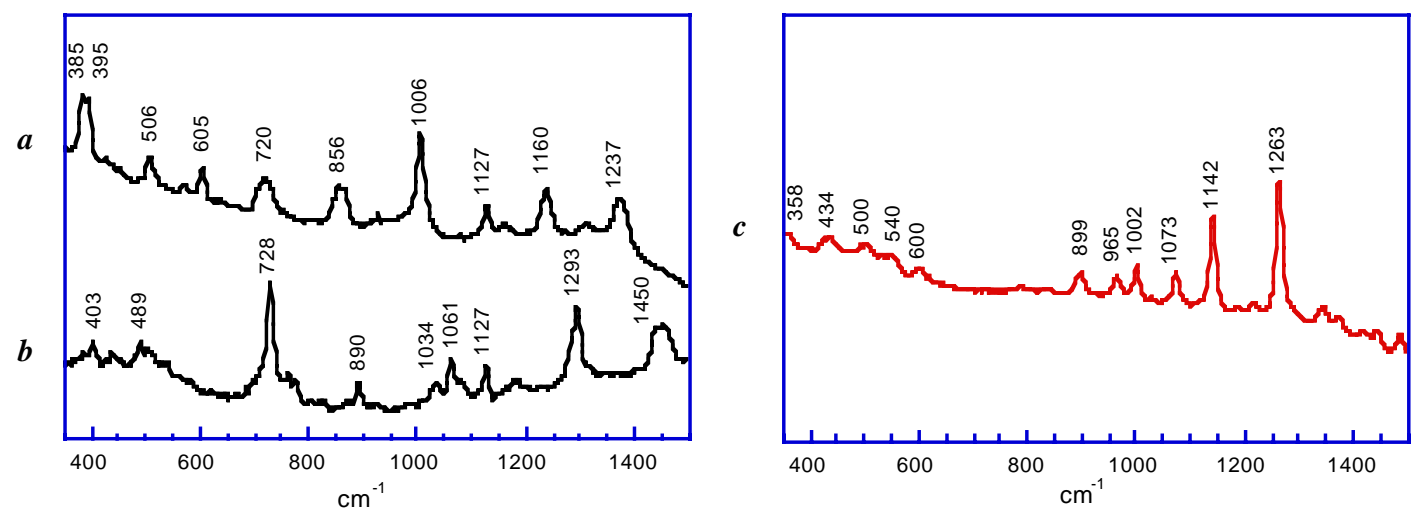

Figure 3. Raman spectra $(500 \mathrm{~mW}$ diode laser, $\lambda=785 \mathrm{~nm}$, integration time $=30 \mathrm{sec})$ of substrates containing resorcinarene 2. $a$, large Au nanoparticles encapsulated by $\mathbf{2} . b, 2$ in bulk polycrystalline phase. $c$, charge-stabilized $\mathrm{Au}$ nanoparticles with an overlayer of 2.

A sample in which 2 was physisorbed to the surface of charge-stabilized Au colloid was prepared and examined by SERS for comparison (see Figure 3c). 15-20 nm Au particles generated by citrate reduction ${ }^{14}$ were precipitated onto a glass slide and coated with a thin film of 2 . This sample produced completely different Raman scattering frequencies and intensity patterns than those generated by the encapsulated nanoparticles. We have recently ascertained that these Raman signals originate from adsorbates other than 2 , but interestingly, none of these were observed prior to addition of $\mathbf{2}$, suggesting a surface dielectric effect. ${ }^{15}$ Nevertheless, this result also indicates that the signals in Figure 3 a are the result of direct chemisorption of 2 to the nanoparticle surface.
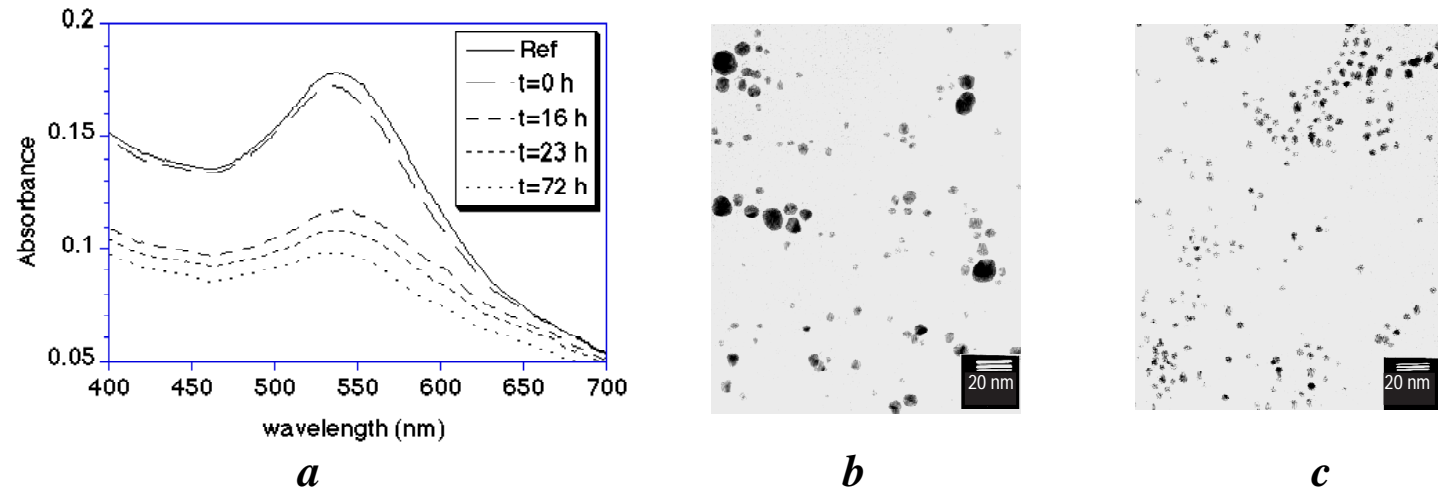

Figure 4. $a$ : UV-vis absorption spectra of 3-15 $\mathrm{nm}$ Au particles dispersed into a $0.1 \mathrm{mM}$ solution of resorcinarene 3 (Ref). Treatment with one equivalent of dodecanethiol resulted in a loss of absorbance over time $(t=0-72 \mathrm{~h}) . b, c$ : Transmission electron micrographs of Au nanoparticle dispersion before $(b)$ and after $(c)$ addition of dodecanethiol.

Displacement of the resorcinarenes by the more strongly adsorbing dodecanethiol (C12SH) resulted in the precipitation of the larger nanoparticles. A solution containing 3-15 nm Au particles stabilized in a $0.1 \mathrm{mM}$ solution of $\mathbf{3}$ was treated with one molar equivalent of $\mathrm{C} 12 \mathrm{SH}$ and was observed to lighten over time, as quantified by UV-vis absorption spectroscopy (see Figure 4a). 
TEM analysis of the dispersions before and one week after $\mathrm{C} 12 \mathrm{SH}$ addition indicate that particles $>5 \mathrm{~nm}$ in diameter were no longer present, correlating the loss of color to the agglomeration and precipitation of the larger particles (see Figures 4b,c). This demonstrates the resorcinarenes' exceptional ability to prevent the larger nanoparticles from aggregating in solution.

Although resorcinarenes 1-3 have shown potential for maintaining large Au nanoparticles in a dispersed state, attempts to isolate the encapsulated nanoparticles from solution were unsuccessful. Purification by repeated precipitation or by chromatography resulted in extensive degradation. Excessive dilution of the surfactant also led to the gradual precipitation of the resorcinareneencapsulated particles, suggesting facile chemical exchange. Appropriate modification of the resorcinarene headgroup can increase the robustness of the encapsulation shell, so that chargeneutral nanoparticles can be isolated and characterized as discrete chemical entities.

To this end, we have recently synthesized tetrakis(phenylphosphinyl)resorcinarene $\mathbf{5}^{\mathbf{1 6}}$ and have found it to be an excellent surfactant for large $\mathrm{Au}$ nanoparticles. The four phosphorus(III) atoms on the headgroup of $\mathbf{5}$ bond more strongly to the Au surface than the ether oxygens, and are also able to act synergetically for exceptionally strong chemisorption. A polydisperse mixture of Au nanoparticles was generated as described above and captured in a $0.1 \mathrm{mM}$ mesitylene solution of 5. These encapsulated particles could be separated from excess surfactant by preparative gelpermeation chromatography (GPC) and concentrated to dryness without degradation. Analytical GPC analysis using a Waters Styragel HR 4E column $\left(\mathrm{CHCl}_{3}\right)$ identified a high molecular-weight species with strong absorptivities at both 254 and $540 \mathrm{~nm}$; TEM analysis of this fraction revealed large Au nanoparticles (see Figure 5).

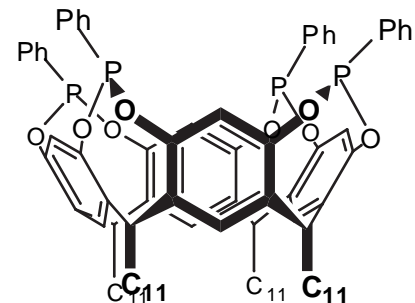

5

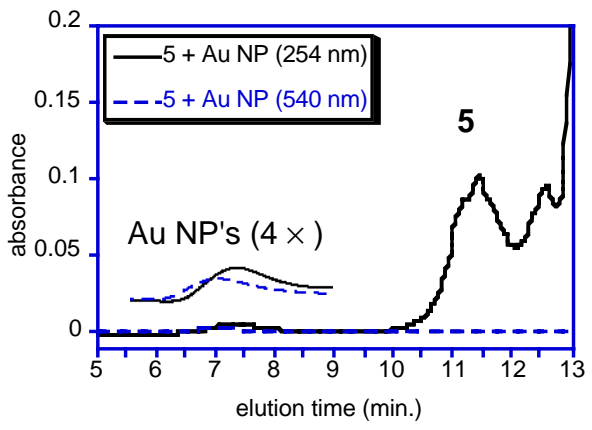

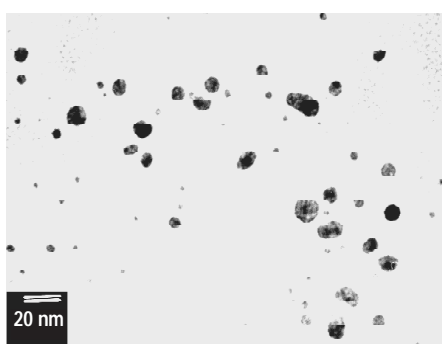

Figure 5. Large $\mathrm{Au}$ nanoparticles encapsulated by resorcinarene 5 (left) were isolated by gel permeation chromatography (middle) and visualized by transmission electron microscopy (right).

\section{CONCLUSIONS}

We have shown that gold nanoparticles up to $20 \mathrm{~nm}$ in diameter can be successfully stabilized by resorcinarene-based surfactants. The chemisorption of resorcinarenes 1-3 to the Au surface is most likely mediated by multidentate Au-O interactions, which were of sufficient strength to prevent agglomeration as demonstrated by TEM and UV-vis absorption spectroscopy. This specific mode of chemisorption is supported by the inability of methylene-bridged resorcinarene 4 to stabilize $\mathrm{Au}$ nanoparticles, as well as by surface-enhanced Raman spectroscopy. The displacement of resorcinarenes by dodecanethiol resulted in a marked precipitation of the larger $\mathrm{Au}$ nanoparticles, indicating that tailgroup mobility is an important factor for maintaining the encapsulated nanoparticles in a dispersed state. The surface bonding of resorcinarenes 1-3 is not strong enough to enable isolation of the encapsulated particles, but functionalizing the surfactant with highly chemisorptive phosphorus groups addresses this limitation. With this modification, we anticipate that encapsulation of $\mathrm{Au}$ nanoparticles by resorcinarene-based surfactants will provide a significant advance in the controlled fabrication of nanoparticle-based devices.

\section{ACKNOWLEDGEMENTS}

The authors gratefully acknowledge financial support from DARPA (DAAH04-96-1-0437: K.B.S., R.P.A.), the Purdue Research Foundation (fellowship to S.V.P.) and the Research 
Corporation Innovation Award program (RI-0333: A.W.), and to Prof. Michael Weaver for helpful discussions and the use of his laser system.

\section{REFERENCES}

${ }^{1}$ (a) Andres, R. P.; Bein, T.; Dorogi, M.; Feng, S.; Henderson, J. I.; Kubiak, C. P.; Mahoney, W.; Osifchin, R. G.; Reifenberger, R. Science 1996, 272, 1323-25. (b) Andres, R. P.; Bielefeld, J. D.; Henderson, J. I.; Janes, D. B.; Kolagunta, V. R.; Kubiak, C. P.; Mahoney, W. J.; Osifchin, R. G. Science 1996, 273, 1690-93. (c) Service, R. F. Science 1996, 271, 920-22. (d) Alivisatos, A. P. Science 1996, 271, 933-37. (e) Klein, D. L.; Roth, R.; Lim, A. K. L.; Alivisatos, A. P.; McEuen, P. L. Nature 1997, 389, 699-701. (f) Feldheim, D. L.; Keating, C. D. Chem. Soc. Rev. 1998, 27, 1-12. (g) Smith, C. G. Science 1999, 284, 274.

${ }^{2}$ Osifchin found that alkanethiol-passivated gold clusters with diameters larger than $\sim 7 \mathrm{~nm}$ do not form stable colloidal solutions in hydrocarbon solvents: Osifchin, R. G. PhD Thesis, Purdue University, 1994. The flocculation of alkanethiol-coated $\mathrm{Au}$ nanoclusters with diameters larger than $10 \mathrm{~nm}$ has also been studied in some detail as a function of surfactant: Weisbecker, C. S.; Merritt, M. V.; Whitesides, G. M. Langmuir 1996, 12, 3763-72.

${ }^{3}$ Israelachvili, J. Intermolecular and Surface Forces; 2nd ed.; Academic Press: New York, 1992. Chapter 10.

${ }^{4}$ Timmerman, P.; Verboom, W.; Reinhoudt, D. N. Tetrahedron 1996, 52, 2663-704.

${ }^{5}$ Whitesides, G. M.; Simanek, E. E.; P., M. J.; Seto, C. T.; Chin, D. N.; Mammen, M.; Gordon, D. M. Acc. Chem. Res. 1995, 28, 37-44.

${ }^{6}$ Parts of this section have been described in a recently submitted publication: Stavens, K. B.; Pusztay, S. V.; Zou, S.; Andres, R. P.; Wei, A. Langmuir, in press.

${ }^{7}$ Chao, L. C., Andres, R. P. J. Colloid Interface Sci. 1994, 165, $290-95$.

${ }^{8}$ (a) Aoyama, Y.; Tanaka, Y.; Sugahara, S. J. Am. Chem. Soc. 1989, 111, 5397-404. (b) van Velzen, E. U. T.; Engbersen, J. F. J.; Reinhoudt, D. N. Synthesis 1995, 989-97.

${ }^{9}$ Mahoney, W. J. and Andres, R. P. Materials Sci. Eng. 1995, A204, 160.

${ }^{10}$ (a) Cram, D. J. Angew. Chem. Int. Ed. Engl. 1986, 25, 1039-1134. (b) Lehn, J.-M. Supramolecular Chemistry: Concepts and Perspectives; VCH Publishers: New York, NY, 1995.

${ }^{11}$ Masel, R. I. Principles of Adsorption and Reaction on Solid Surfaces, John Wiley and Sons, New York, 1996, Chapter 3.

12 (a) Freeman, R. G.; Grabar, K. C.; Allison, K. J.; Bright, R. M.; Davis, J. A.; Guthrie, A. P.; Hommer, M. B.; Jackson, M. A.; Smith, P. C.; Walter, D. G.; Natan, M. J. Science 1995, 267, 1629-32. (b) Freeman, R. G.; Hommer, M. B.; Grabar, K. C.; Jackson, M. A.; Natan, M. J. J. Phys. Chem. 1996, 100, 718-24. (c) Vo-Dinh, T. Trends Anal. Chem. 1998, 17, 557-82.

${ }^{13}$ Weaver, M. J., Zou, S. in Advances in Spectroscopy 26, John Wiley and Sons, Chichester, 1998, Chapter 5, and references therein.

${ }^{14}$ (a) Turkevich, J.; Stevenson, P. C.; Hillier, J. Disc. Farad. Soc. 1951, 11, 55-75. (b) Frens, G. Nature Phys. Sci. 1973, 241, 20-22. (c) Handley, D. A. Colloidal Gold: Principles, Methods, and Applications; Hayat, M. A., Ed.; Academic Press: San Diego, 1989; Vol. 1, pp 14-32.

${ }_{15}$ These signals presumably arise from citrate ions and/or their degradation byproducts adsorbed to the gold surface, although their frequencies do not overlap with those from crystalline sodium citrate or from citrate adsorbed onto Ag colloid (Blatchford, C.G.; Siiman, O.; Kerker, M. J. Phys. Chem. 1983, 87, 2503-08). These authors have suggested that the absorbate spectra and peak intensities may be strongly affected by the surface potential and by variable aggregation states.

${ }^{16}$ Xu, W.; Rourke, J. P.; Vittal, J. J.; Puddephatt, R. J. Inorg. Chem. 1995, 34, 323-29. 